\title{
Looking Inside-Out: The impact of Corporate Environmental Responsibility on Employee's Organizational Citizenship Behavior
}

\author{
${ }^{1}$ Sílvia Lopes, ${ }^{2}$ Marta Ferreira, ${ }^{3}$ Luis D. Torres, ${ }^{4}$ Ana Sabino, ${ }^{5}$ Luís Pimentel \\ ${ }^{1}$ CICPSI, Faculdade de Psicologia, Universidade de Lisboa, Portugal \\ Universidade Europeia, Lisbon, Portugal ${ }^{, 2}$ Universidade Europeia, Lisbon, Portugal, ${ }^{3}$ Nottingham University \\ Business School China, ${ }^{4}$ Universidade Europeia, Lisbon, Portugal,${ }^{5}$ Universidade Europeia, Lisbon, Portugal
}

\begin{abstract}
Climate change is one of the most pressing environmental, economic and social issues for humanity nowadays. As such, society is becoming increasingly more conscious and concerned about the environmental impacts of their activities and, particularly, requesting the corporate sector to do the same. In this cross-sectional study design, we studied the relationship between employees' perceptions of corporate environmental responsibility (CER), employee environmental commitment, organizational citizenship behavior towards the environment (OCBE), and company size. This research involved a sample of Portuguese active population $(n=156)$, and the sample size was reduced to those individuals who were familiar with the practices of CER in the company where they work $(n=116)$. Results showed that regardless of the company size - i.e., small and medium-sized enterprises (SMEs) vs large companies - no significant differences were observed in the employees' perception of CER practices. There was a positive and significant relationship between the perception of CER practices and OCBE, as well as environmental commitment was observed as having a mediating role in the relationship between the employees' perception of CER practices and OCBE. In conclusion, employees' perception of CER practices contributes significantly to OCBE. In addition, commitment with CER seems to be a mechanism that contributes to fully explain the relationship between the employees' perception of CER practices and OCBE. The implications of these results will be discussed at the conference.
\end{abstract}

Keywords: Corporate social responsibility; corporate environmental responsibility; sustainable development; organizational citizenship behavior; environmental commitment. 